\title{
openheart Physical exercise and quantitative lower limb collateral function
}

\author{
Michael Stoller, David Stoller, Christian Seiler
}

To cite: Stoller M, Stoller D, Seiler C. Physical exercise and quantitative lower limb collateral function. Open Heart 2016;3:e000355. doi:10.1136/openhrt-2015000355

MS and DS contributed equally.

Received 29 October 2015 Revised 11 January 2016 Accepted 14 January 2016

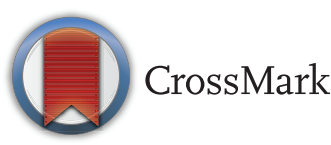

Department of Cardiology, University Hospital, Bern, Switzerland

Correspondence to Professor Christian Seiler; christian.seiler@insel.ch

\section{ABSTRACT}

Objective: This study tested the hypothesis that global physical activity and physical performance parameters are directly related to invasively obtained left superficial femoral artery (SFA) collateral flow index (CFI).

Background: So far, the association between different measures of physical exercise activity and quantitative lower limb collateral function has not been investigated.

Methods: The primary study end point was pressurederived CFI as obtained during a 3 min left SFA balloon occlusion. CFI is the ratio of simultaneously recorded mean SFA distal occlusive pressure divided by mean aortic pressure, both subtracted by central venous pressure. As independent variables, the items of the Global Physical Activity Questionnaire (GPAQ) and physical exercise performance (maximal workload in watts) as achieved during a bicycle or treadmill exercise test were determined. The secondary study end point was transcutaneous left calf partial oxygen pressure $\left(\mathrm{PO}_{2}\right.$ in $\left.\mathrm{mm} \mathrm{Hg}\right)$ divided by transcutaneous $\mathrm{PO}_{2}$ at a non-ischaemic reference site as obtained simultaneously to $\mathrm{CFI}$ measurement.

Results: Of the 110 study patients undergoing diagnostic coronary angiography, 79 belonged to the group without and 31 with engagement in regular intensive leisure time physical activity according to GPAQ. Left SFA CFI tended to be lower in the group without than with intensive leisure time physical activity: $0.514 \pm 0.141$ vs $0.560 \pm 0.184(p=0.0566)$. Transcutaneous $\mathrm{PO}_{2}$ index was associated with simultaneous left SFA CFI: CFI $=018+0.57 \mathrm{PO}_{2}$ index; $p<0.0001$. Maximal physical workload was directly associated with left SFA CFI: CFI $=0.40+0.0009$ maximal workload; $p=0.0044$.

Conclusions: Quantitative left SFA collateral function is directly reflected by maximal physical workload as achieved during an exercise test.

Trial registration number: NCT002063347.

\section{INTRODUCTION}

Cardiovascular diseases remain the number one cause of death globally. ${ }^{1}$ Among them, lower limb peripheral artery disease (PAD) is a leading cause of morbidity, and has a prevalence of $15-20 \%$ in patients older than 65 years of age. ${ }^{2}$ The primary clinical end point of PAD, critical limb ischaemia (rest

\section{KEY QUESTIONS}

What is already known about this subject?

- In patients with coronary artery disease, a direct association between the degree of leisure time physical activity and quantitative coronary collateral function has been shown, the relation of which appears to be causal.

What does this study add?

- This clinical study finds a similar direct relation between the level of physical fitness as obtained during a stress test, and quantitative lower limb collateral function.

How might this impact on clinical practice?

- This finding further supports counselling patients with peripheral artery disease to engage in regular physical exercise as primary treatment.

pain, non-healing wounds or gangrene, ankle or toe arterial pressure $<30 \mathrm{~mm} \mathrm{Hg}$ ) as observed over a 5-year period, occurs at a rate of $1.3 \%$, that is, it develops in approximately every 10th patient with PAD. ${ }^{2}$ That critical limb ischaemia develops relatively rarely is biologically related to a welldeveloped collateral function in the presence as well as absence of PAD. ${ }^{3}{ }^{4}$ In humans, lower limb collateral function has not been quantitatively measured until very recently, when a small study has shown that in the absence of PAD, superficial femoral artery (SFA) balloon occlusion during $5 \mathrm{~min}$ is painless at rest, and that the flow via anastomoses to the occluded SFA amounts to approximately $55 \%$ of the flow during SFA patency. ${ }^{4}$ During continued SFA occlusion, and in response to foot exercise, $89 \%$ of patients in that study developed claudication until occlusion was stopped at $10 \mathrm{~min}$. Experimental investigations have documented arteriogenic efficacy of physical exercise in the hind limb. ${ }^{5}$ Physical exercise training programmes have been shown to alleviate claudication in PAD, and meta-analyses have found an extended walking distance until claudication onset. $^{6-9}$ However, as 
exemplified, imprecise measures of therapeutic effects in clinical PAD studies have been employed, such as the mentioned walking distance, decrease of ulcer size, resting leg pain, ankle-brachial index, digital subtraction angiography, and quality-of-life questionnaires. Considering a cross-sectional pathogenic rather than longitudinal therapeutic view, a recent investigation using SFA MRI has-not unexpectedly-found that SFA occlusion and a low collateral vessel score are associated with poor functional walking performance. ${ }^{10}$

In this context of lacking evidence of an association between physical exercise and quantitative lower limb collateral function, the present prospective crosssectional study tested the hypothesis that measures of global physical activity and physical performance are directly related to invasively obtained SFA collateral flow index.

\section{METHODS}

\section{Study design and patients}

This was a prospective observational study in 110 patients undergoing coronary angiography for diagnostic purposes in the context of chest pain. The primary study end point was pressure-derived collateral flow index (CFI; see below for calculation) as obtained during a 3 min left SFA balloon occlusion (figure 1). The secondary study end point was the simultaneously measured transcutaneous left calf partial oxygen pressure $\left(\mathrm{PO}_{2}\right.$ in $\left.\mathrm{mm} \mathrm{Hg}\right)$ divided by transcutaneous $\mathrm{PO}_{2}$ at a non-ischaemic reference site (figure 2). As independent variables, the items of the Global Physical Activity Questionnaire $\left(\mathrm{GPAQ}^{11}\right)$ and physical exercise performance as obtained during a bicycle or treadmill exercise test were determined. Criteria for study inclusion were age $>18$ years, written informed consent to participate in the study, 0-vessel to 3-vessel chronic stable coronary artery disease $(\mathrm{CAD})$, and a complete data set with regard to the primary study end point, to the completed GPAQ and maximal workload in the physical exercise test. Exclusion criteria were acute coronary syndrome, previous myocardial infarction in the vascular region undergoing coronary CFI measurement, severe hepatic or renal failure (creatinine clearance $<15 \mathrm{~mL} / \mathrm{min}$ / $1.73 \mathrm{~m}^{2}$ ). The study was approved by the ethics committee of the Kanton of Bern, Switzerland, and all patients gave written informed consent to participate.

\section{Cardiac catheterisation and coronary angiography}

Patients underwent left heart catheterisation and coronary angiography for diagnostic purposes from the right femoral artery approach via a $65 \mathrm{~cm}$-long $8 \mathrm{~F}$ introducer sheath. Biplane left ventriculography was performed followed by coronary angiography. Coronary artery stenoses were assessed quantitatively as per cent diameter reduction using the guiding catheter for calibration. Aortic pressure $\left(\mathrm{P}_{\mathrm{ao}}\right)$ was obtained via a $6 \mathrm{~F}$ or $8 \mathrm{~F}$ arterial guiding catheter. Central venous pressure (CVP) was measured as right atrial pressure via the right femoral vein. CFI was also determined in one of the coronary arteries, whereby it was measured and calculated as subsequently described for the left SFA.

\section{Invasive collateral assessment}

\section{Primary study end point}

Arterial occlusive collateral flow relative to normal antegrade flow through the non-occluded artery $(\mathrm{CFI})$ in the left SFA was determined using arterial pressure measurements. A 0.014 inch pressure sensor angioplasty guide wire (Pressure Wire, St Jude Medical, Eschborn, Germany) was calibrated, advanced through and just outside the guiding catheter, where equalisation with the guide catheter pressure was performed and positioned in the distal part of the SFA (figure 1). CFI was determined by simultaneous measurement of mean aortic pressure $\left(\mathrm{P}_{\mathrm{ao}}, \mathrm{mm} \mathrm{Hg}\right)$, the distal SFA pressure during balloon occlusion $\left(\mathrm{P}_{\mathrm{occl}}, \mathrm{mm} \mathrm{Hg}\right)$, and the CVP $(\mathrm{mm} \mathrm{Hg})$, as obtained during the last $30 \mathrm{~s}$ of the $3 \mathrm{~min}$ SFA balloon occlusion (figure 1). Left SFA CFI was calculated as $\left(\mathrm{P}_{\mathrm{occl}^{-}} \mathrm{CVP}\right)$ divided by $\left(\mathrm{P}_{\mathrm{ao}}-\mathrm{CVP}\right) .{ }^{4}{ }^{12}$ The accuracy of pressure-derived coronary CFI measurements in comparison with ECG signs of myocardial ischaemia during occlusion, and to absolute myocardial perfusion measurements, has been documented previously. ${ }^{12-14}$ CFI measurements in the SFA have been evaluated in comparison with simultaneously obtained arterial Doppler measurements in a small group of patients. $^{4}$

\section{Secondary study end point}

The ratio of ischaemic to non-ischaemic transcutaneous $\mathrm{PO}_{2}$ (SFA transcutaneous $\mathrm{PO}_{2}$ index; figure 2) was obtained simultaneously to SFA CFI measurement. Transcutaneous $\mathrm{PO}_{2}$ measurements were performed using the TCM 400 Monitoring System (RADIOMETER RSCH GmbH, Thalwil, Switzerland). SFA transcutaneous $\mathrm{PO}_{2}$ index $(\mathrm{mm} \mathrm{Hg} / \mathrm{mm} \mathrm{Hg})$ was calculated at the through value at the ischaemic site (left anteromedial calf) divided by the reference value at the nonischaemic site (left lower abdomen).

\section{Invasive study protocol}

Immediately before the invasive exam, transcutaneous $\mathrm{PO}_{2}$ sensors were applied after skin preparation. Skin areas with large veins, skin defects or scars were avoided for sensor placement. The controlled heating element around the sensor was adjusted to $44^{\circ} \mathrm{C}$, and sensor readings were allowed to stabilise for at least $20 \mathrm{~min}$ before the relevant measures were taken. The $\mathrm{PO}_{2}$ sensors were placed at the left anteromedial calf, halfway below the knee (ischaemic site), and at the lower left abdomen (non-ischaemic reference site). Also, before the diagnostic examination, two puffs of oral isosorbidedinitrate were given. Following diagnostic coronary angiography, and at the start of the invasive study procedure, all patients received 10000 units of heparin 

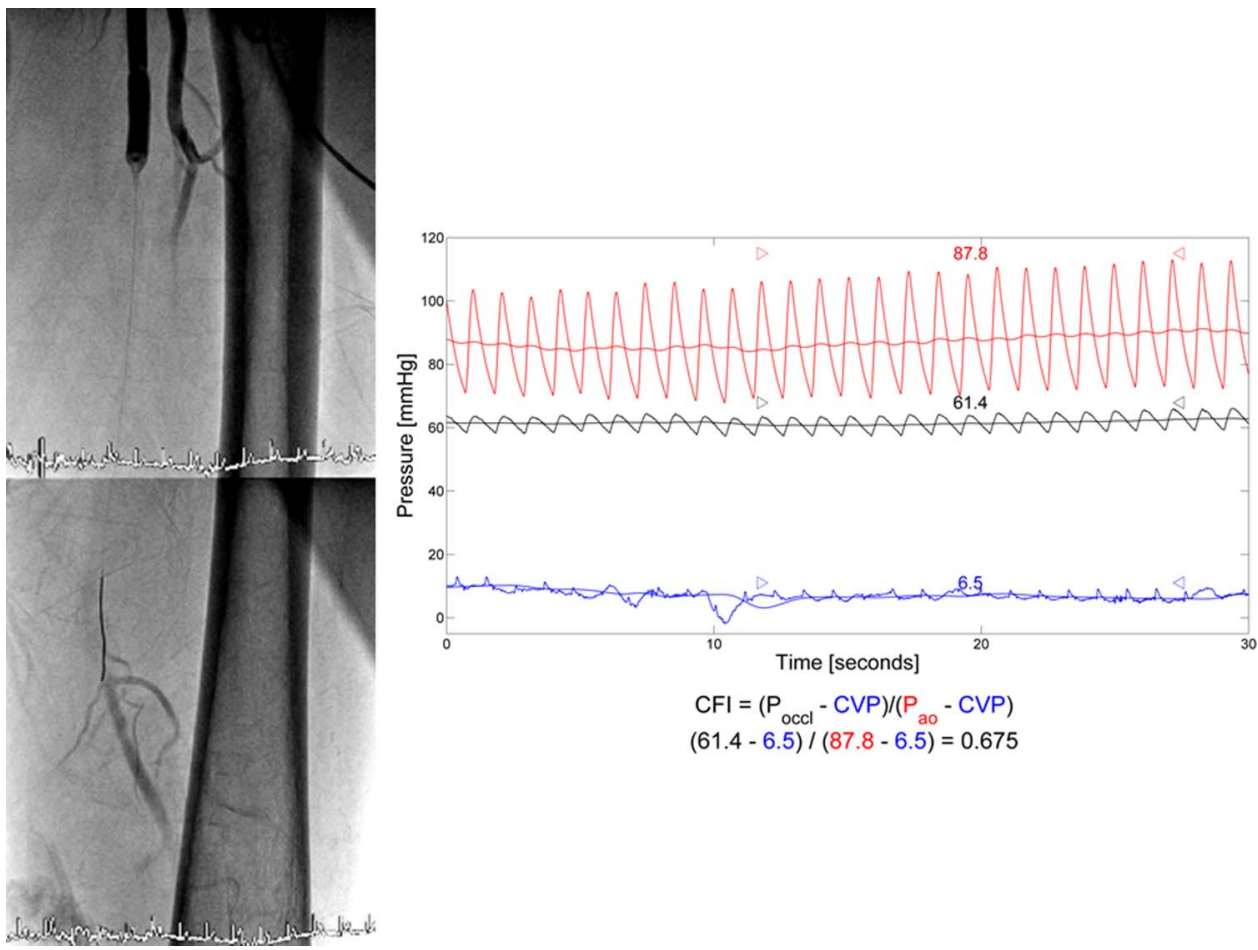

Figure 1 Angiographic image of the angioplasty-balloon-occluded left superficial femoral artery (SFA; left panel), which is partly filled with radiographic contrast medium via collaterals. Distal to the occlusive balloon, a pressure-sensor-guide wire is placed with the sensor located at the junction between the opaque and the more transparent part of the wire. During the imaged SFA occlusion, the following phasic and mean pressure curves are simultaneously recorded for SFA collateral flow index (CFI) measurement (right panel): aortic pressure $\left(P_{\text {ao }}\right.$, red curve), SFA distal occlusive pressure ( $P_{\text {occl }}$, black curve), and central venous pressure (CVP, black curve). SFA CFI=( $\left.\mathrm{P}_{\text {occl}}-\mathrm{CVP}\right) /\left(\mathrm{P}_{\mathrm{ao}}-\mathrm{CVP}\right)$.

intravenously. The coronary artery undergoing CFI measurements was chosen on the basis of ease of access in case of a non-stenotic vessel, or the presence of a stenotic lesion requiring percutaneous coronary intervention. Coronary fractional flow reserve (FFR) was obtained prior to coronary CFI. FFR was determined

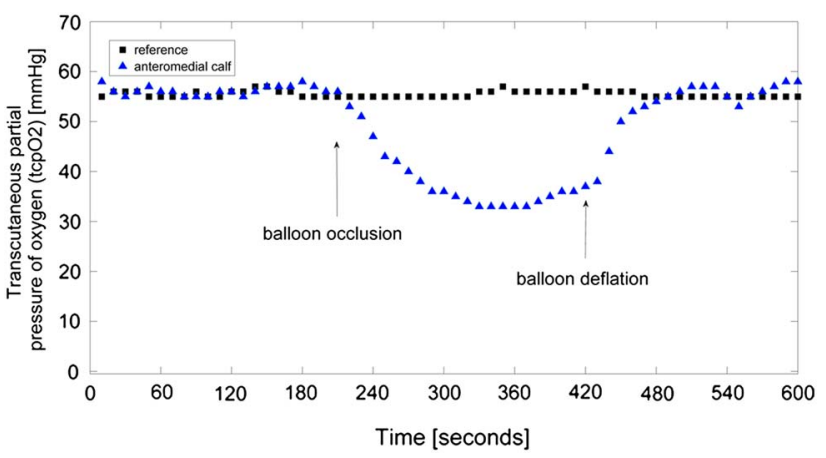

Figure 2 Simultaneously recorded transcutaneous partial oxygen pressure $\left(\mathrm{PO}_{2}\right)$ before and during a $3 \mathrm{~min}$ left superficial femoral artery (SFA) balloon occlusion. Transcutaneous $\mathrm{PO}_{2}$ sensors are located at the ischaemic site (anteromedial calf; blue symbols) and at the non-ischaemic reference site (lower left abdomen; black symbols). The transcutaneous $\mathrm{PO}_{2}$ index is equal to $33 \mathrm{~mm} \mathrm{Hg} / 57 \mathrm{~mm} \mathrm{Hg}=0.579$. with the pressure guide wire positioned distally in the non-occluded coronary artery of interest using an intracoronary bolus of approximately $70 \mu \mathrm{g}$ adenosine for hyperaemia induction: FFR=distal coronary pressure divided by $\mathrm{P}_{\mathrm{ao}}$. For coronary CFI measurement, an adequately sized monorail angioplasty balloon catheter (diameter ranging $2.5-5 \mathrm{~mm}$ ) was positioned in the proximal part of the vessel, while the pressure guide wire remained distally. During vessel occlusion, simultaneous coronary $\mathrm{P}_{\text {occl }}, \mathrm{P}_{\mathrm{ao}}$ and CVP were obtained for the calculation of CFI. Right atrial pressure was taken as CVP via a $5 \mathrm{~F}$ pigtail catheter. Following coronary CFI measurement, left SFA CFI measurement was performed by crossover technique via the right femoral artery $8 \mathrm{~F}$ introducer sheath using an over-the-wire 10-12 mm angioplasty balloon ( 3 min occlusion). CVP was taken at the right atrium. Simultaneously, transcutaneous $\mathrm{PO}_{2}$ measurements were recorded. The arterial balloon occlusion occurred at an inflation pressure of 1-2 atmospheres. Complete arterial occlusion was established by angiography. Immediately following the CFI measurements, vessel integrity was tested by angiography.

\section{Physical exercise test}

Data from a recent treadmill or bicycle exercise ECG test, as performed for diagnostic purposes in the context of chest pain, was obtained from the hospital charts. 
The independent (and universally available) variable employed for this study was maximal physical workload in watts.

\section{Global Physical Activity Questionnaire}

Using the structured GPAQ information was gained on the time spent performing different types of physical activity in a typical week. ${ }^{15}$ The questionnaire consists of 16 questions covering the intensity level and estimated time spent during work and leisure time physical activity, as well as during travel to and from places using physical work. According to the patient's mother tongue, the German or French language version of the questionnaire was mailed to the patients following the invasive SFA CFI assessment. The GPAQ has been extensively validated. ${ }^{1617}$

\section{Statistical analysis}

For the purpose of data presentation, two study groups, that is, with absence or presence of intensive leisure time physical activity were formed based on the information gained from the GPAQ. Between-group comparison of continuous demographic, clinical, angiographic, haemodynamic variables and CFI data was performed by an unpaired Student $t$ test. A $\chi^{2}$ test was used for comparison of categorical variables among the study groups. Linear regression analysis was performed for testing associations between independent variables (transcutaneous $\mathrm{PO}_{2}$ index and maximal workload achieved during exercise test) and the dependent primary study end point (left SFA CFI). Linear regression, and Bland-Altman bias and limit of agreement (LOA: bias \pm $1.96 \mathrm{SDs}$ ) were used to estimate the agreement between the different collateral function modalities. Statistical significance was defined at a p-level $<0.05$. Continuous variables are given as mean and $\mathrm{SD}$.

\section{RESULTS}

The 110 patients with an entire data set of SFA CFI, physical exercise test maximal workload, and completed GPAQ were recruited from a total of 143 patients with invasive collateral function measurements. Of these, 129 had undergone SFA CFI measurement; in 14/143, obtaining the primary end point was not possible due to technical reasons; 30 patients with SFA CFI were included from a previous study on quantitative peripheral collateral function. ${ }^{44}$ A total of 115 GPAQs were completed (response rate $=80 \%$ ). The majority of 79/ 110 patients belonged to the group without a history of intensive leisure time physical activity, 31/110 patients reportedly engaged in intensive leisure time physical activity (table 1 ).

\section{Patient characteristics and clinical data}

There were no statistically significant differences between the groups regarding age, rate of male gender, weight, height, body mass index, occurrence of cardiovascular risk factors and intake of cardiovascular drugs (table 1).

\section{Haemodynamic and physical exercise data}

Mean systemic arterial blood pressure, heart rate, left ventricular ejection fraction, and left ventricular end-diastolic pressure were similar between the groups (table 2). The duration between the invasive exam with SFA CFI measurement and the physical exercise test was not statistically different between the groups. Patients who did not report

Table 1 Patient characteristics and clinical data

\begin{tabular}{lll}
\hline & $\begin{array}{l}\text { No intensive leisure } \\
\text { time physical activity }\end{array}$ & $\begin{array}{l}\text { Intensive leisure } \\
\text { time physical activity }\end{array}$ \\
\hline Number of patients & 79 & 31 \\
Age (years) & $65 \pm 9$ & $64 \pm 11$ \\
Male gender (\%) & $57(72)$ & $31(89)$ \\
Weight (kg) & $82 \pm 12$ & $82 \pm 14$ \\
Height (cm) & $172 \pm 10$ & $174 \pm 9$ \\
Body mass index (kg/m $\left.{ }^{2}\right)$ & $28 \pm 4$ & $27 \pm 4$ \\
Current smoking (\%) & $13(17)$ & $5(15)$ \\
Dyslipidaemia (\%) & $65(82)$ & $24(76)$ \\
Hypertension (\%) & $60(76)$ & $22(71)$ \\
Family history for CAD (\%) & $32(40)$ & $12(39)$ \\
Diabetes mellitus (\%) & $18(23)$ & $7(24)$ \\
Acetylsalicylic acid (\%) & $61(77)$ & $21(67)$ \\
$\beta$-blockers (\%) & $38(44)$ & $14(45)$ \\
Calcium antagonists (\%) & $14(18)$ & $5(15)$ \\
ACE-inhibitor/ARB (\%) & $51(64)$ & $14(45)$ \\
Diuretics (\%) & $23(29)$ & $7(21)$ \\
Nitrates (\%) & $7(9)$ & $1(3)$ \\
Statin (\%) & $56(71)$ & $19(61)$ \\
ARB, angiotensin receptor blocker; CAD, coronary artery disease. & 0.73 \\
\end{tabular}


Table 2 Haemodynamic, physical exercise, coronary stenosis, and collateral function data

\begin{tabular}{lllc}
\hline & $\begin{array}{l}\text { No intensive leisure time physical } \\
\text { activity }\end{array}$ & $\begin{array}{l}\text { Intensive leisure time physical } \\
\text { activity }\end{array}$ & p Value \\
\hline Mean blood pressure (mm Hg) & $95 \pm 17$ & $96 \pm 15$ & 0.56 \\
Heart rate (bpm) & $72 \pm 14$ & $71 \pm 13$ & 0.68 \\
LV ejection fraction (\%) & $58 \pm 11$ & $60 \pm 8$ & 0.34 \\
LV end-diastolic pressure (mm Hg) & $12 \pm 6$ & $11 \pm 5$ & 0.32 \\
Days between SFA CFI and exercise & $-97 \pm 261$ & $-35 \pm 188$ & 0.29 \\
test & & & 0.0222 \\
Maximal workload (watts) & $138 \pm 43$ & $160 \pm 61$ & 0.0142 \\
Maximal workload (watts/kg) & $1.7 \pm 0.57$ & $2.0 \pm 0.82$ & 0.46 \\
Intensive physical work (\%) & $15(18)$ & $8(26)$ & 0.25 \\
Moderate physical work (\%) & $61(77)$ & $27(88)$ & 0.26 \\
Moderate leisure time physical activity & $58(74)$ & $26(85)$ & 0.92 \\
(\%) & & & 0.0531 \\
Travel by foot or bicycle (\%) & $53(67)$ & $21(68)$ & 0.72 \\
Intensive physical work (min/week) & $127 \pm 416$ & $306 \pm 788$ & 0.62 \\
Moderate physical work (min/week) & $839 \pm 992$ & $910 \pm 953$ & \\
Moderate leisure time physical activity & $209 \pm 233$ & $187 \pm 177$ & 0.0668 \\
('/week) & & & 0.27 \\
Travel by foot or bicycle (min/week) & $184 \pm 244$ & $312 \pm 515$ & $<0.0001$ \\
Total physical activity (min/week) & $1399 \pm 1226$ & $1680 \pm 1299$ & \\
Intensive leisure time physical activity & 0 & $169 \pm 186$ & 0.0553 \\
('/week) & & & 0.0909 \\
Coronary per cent diameter stenosis & $34 \pm 22$ & $26 \pm 21$ & 0.0552 \\
Coronary FFR (mm Hg/mm Hg) & $0.86 \pm 0.14$ & $0.91 \pm 0.07$ & 0.0566 \\
Coronary CFI (mm Hg/mm Hg) & $0.107 \pm 0.083$ & $0.139 \pm 0.091$ & \\
SFA CFI (mm Hg/mm Hg) & $0.514 \pm 0.141$ & $0.560 \pm 0.184$ & \\
\hline CFI, collateral flow index; FFR, fractional flow index; LV, left ventricle/ventricular; SFA, superficial femoral artery. & &
\end{tabular}

engaging in intensive leisure time physical activity achieved a lower maximal workload during the exercise test than did patients of the group with intensive leisure time physical activity (table 2). By definition, patients of the group without intensive leisure time physical activity evaluated

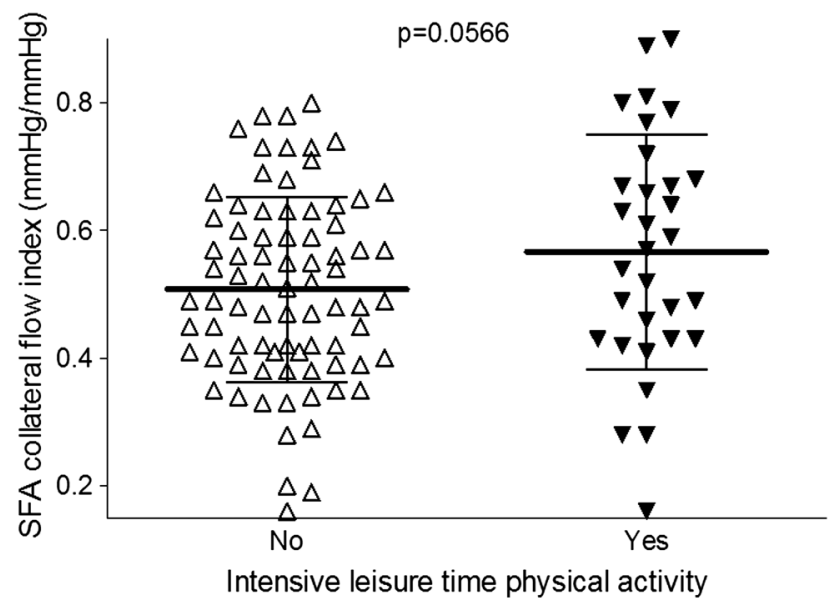

Figure 3 Individual values of left superficial femoral artery (SFA) collateral flow index (CFI; vertical axis) as obtained by the General Physical Activity Questionnaire in the group of patients without (open triangles) and with (closed triangles) intensive leisure time physical activity. The horizontal lines indicate mean $\mathrm{CFI}$ (thick line) $\pm \mathrm{SD}$. the respective duration to be $0 \mathrm{~min} /$ week (table 2). There was a trend towards shorter estimated durations of intensive physical work and travel, by exercise in the group without versus with engagement in intensive leisure time activity (table 2); the other GPAQ items did not differ among the groups.

\section{Coronary stenosis and collateral function data}

Coronary stenosis of the vessel undergoing CFI measurement was more severe both structurally and functionally in the group without versus with reported engagement in intensive leisure time physical activity (table 2). Conversely, coronary CFI revealed a trend towards lower values in the group without versus with engagement in intensive leisure time physical activity. Left SFA CFI tended to be lower in the group without versus with engagement in intensive leisure time physical activity (table 2 and figure 3). The left SFA of all patients was free of stenotic lesions.

Left SFA transcutaneous $\mathrm{PO}_{2}$ index as obtained in 86 patients, was directly and closely associated with simultaneous left SFA CFI (figure 4). Bland-Altman bias between the two methods was +0.065 , and the limits of agreement -0.18 and +0.31 . Maximal workload achieved during a previous physical exercise test correlated directly with left SFA CFI (figure 5). All the other continuous parameters as obtained by the GPAQ (estimates of 

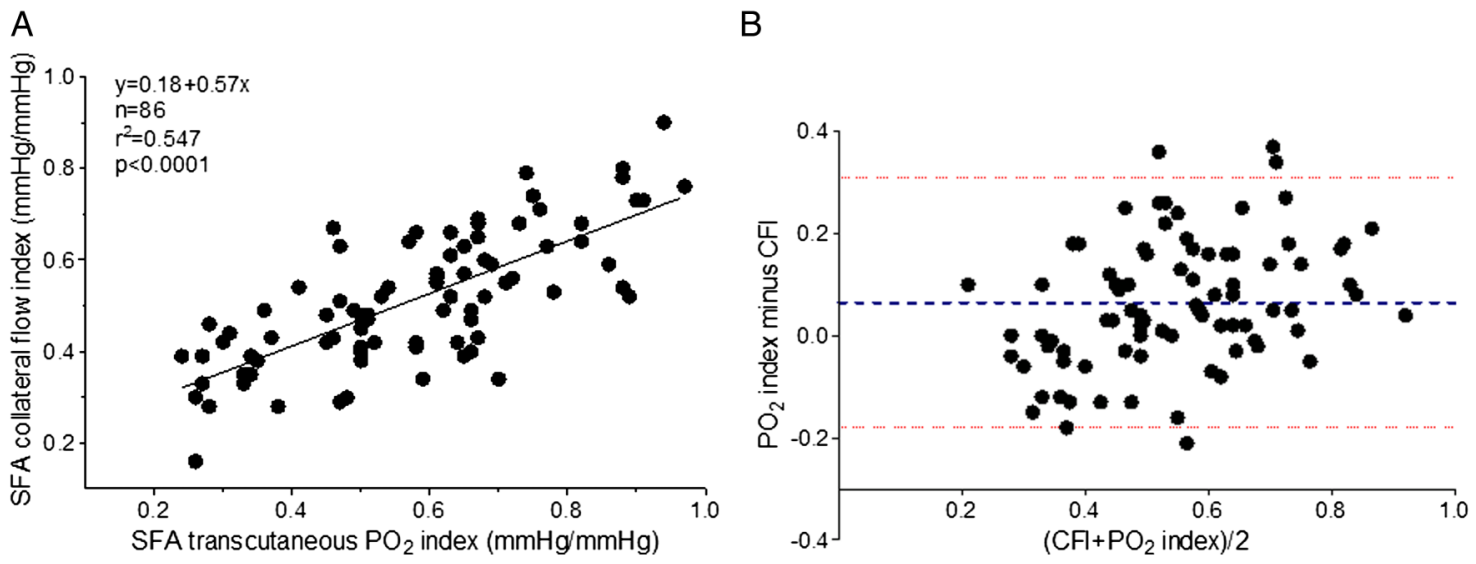

Figure 4 Left panel: Correlation between left superficial femoral artery (SFA) transcutaneous partial oxygen pressure $\left(\mathrm{PO}_{2}\right)$ index (horizontal axis) and the simultaneously obtained left SFA collateral flow index (CFI; vertical axis). Right panel:

Bland-Altman plot of the average between SFA $\mathrm{PO}_{2}$ index and CFI (horizontal axis), and the difference between the two (vertical axis) for each individual point. Blue broken line: level of bias; red broken lines: limits of agreement.

the different work, leisure time and physical travel items in minutes per week; see also table 2) were not associated with SFA CFI.

\section{DISCUSSION}

This cross-sectional study found quantitative, validated, left SFA pre-existent collateral function (CFI without SFA stenoses) to be directly predicted by maximal workload achieved during a physical exercise test. Accordingly, patients reporting intensive leisure time physical activity tended to have a higher SFA CFI than those without such a hobby.

\section{Exercise and collateral function: clinical evidence}

There are currently four treatment modalities reportedly reducing disability and walking distance in intermittent claudication, the most common symptom of lower limb

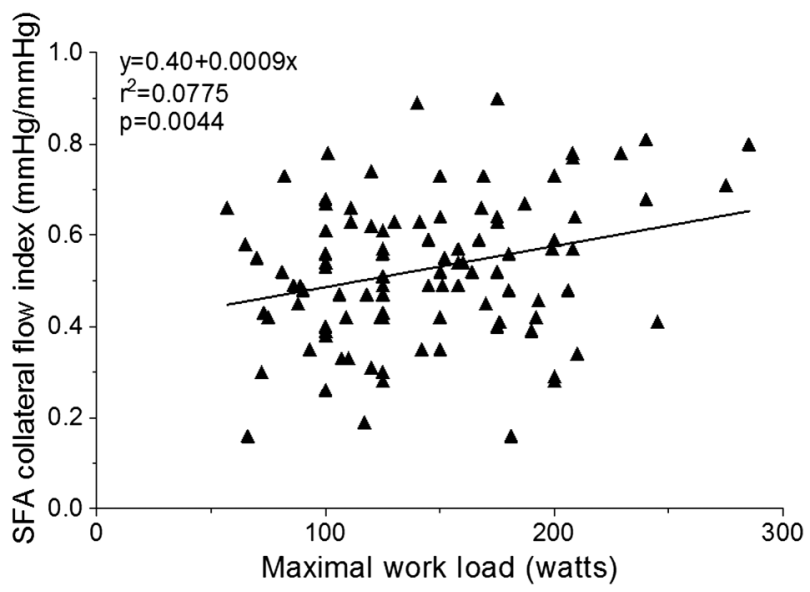

Figure 5 Correlation between maximal workload as achieved during a physical exercise test (treadmill or bicycle; horizontal axis) and left superficial femoral artery (SFA) collateral flow index (CFI; vertical axis).
PAD: physical exercise training, cilostazol, percutaneous intervention of pad, and surgical bypass. ${ }^{18}$ According to expert consensus, ${ }^{19}$ supervised exercise training is the primary treatment modality for patients with intermittent claudication. ${ }^{7} 2021$ Furthermore, the efficacy of even non-supervised physical exercise training appears to be agreed upon. ${ }^{8}$ However, given the frequently employed non-specific clinical end point of walking distance, the mechanism (ischaemic preconditioning, training-induced muscle conditioning, collateral promotion) by which exercise exerts its positive effect remains elusive. In cross-sectional, as opposed to longitudinal PAD studies, the association of exercise training/performance and lower limb collateral circulation has, however, been directly addressed by using walking distance as an end point for exercise performance and not, implicitly, for the collateral circulation, which ought to be determined on its own. For example, using MRI of the SFA and collateral vessel number, McDermott $e t a l^{10}$ found that the 6 min walking distance lost by SFA occlusion (from 1169 with patent SFA to 1031 feet) was overcompensated for by the presence of $\geq 8$ collaterals (1246 vs 1064 feet with 0-3 collaterals). The selection of patients with total SFA occlusion in that study elegantly circumvented the pitfall of a variable influence of different SFA stenoses on collaterals. In analogy, the present study employed an artificial instead of natural SFA occlusion in the absence of SFA stenotic lesions, thus, also correcting for the diverse arteriogenic influence of varying severities of stenosis. Variable severities of stenosis instead of fixed ones (no stenosis or complete occlusion), could actually lead to opposite findings as in the just cited recent study, ${ }^{10}$ that is, a higher collateral degree associated with a reduced prevalence of asymptomatic PAD. ${ }^{22}$ In other words, the collateral circulation is both a benefactor against, and a marker for, the severity of atherosclerosis, the methodological conundrum of which has been resolved in the present study by directly 
measuring the SFA collateral function, and by determining two potential independent predictors, that is, GPAQ items and maximal workload achieved in an exercise test.

Most commonly used questionnaires to assess functional status and general as well as disease-specific quality of life in patients with intermittent claudication do not include GPAQ. ${ }^{23}$ To the best of our knowledge, physical activity questionnaires have not been used so far in the context of lower limb collateral function. In patients with $\mathrm{CAD}$, the level of leisure time physical activity, as scored by a structured but not validated questionnaire, and coronary CFI, have been documented to be directly associated. ${ }^{24}$ In the present investigation, this result could be essentially confirmed, though the current as opposed to the past study population had much less severe stenosis in the coronary artery undergoing CFI measurement. Interestingly, coronary stenosis severity in the CFI vessel tended to be more pronounced among patients without versus with reported leisure time physical activity (see table 2); and nevertheless, coronary CFI was higher among patients engaging in leisure time physical activity. Though the coronary circulation was not in the focus of this investigation, the respective results anchor its main findings. GPAQ has been extensively validated, ${ }^{16}$ and its German version has also been employed in a recent survey on self-reported physical activity across Germany as obtained by a telephone interview. ${ }^{17}$ By comparison, the present study gained GPAQ data by telephone interview only in a minority of approximately $10 \%$ of the entire study group, that is, in those patients who did not answer the first call to fill out the questionnaire. Answering the GPAQ unassisted certainly complicated the questionnaire's part in which total physical activity durations in minutes per week had to be estimated. This is reflected by the study result that only a dichotomous GPAQ item, the grouping variable, 'intensive leisure time physical activity', was mirrored by maximum physical workload.

\section{Maximal workload and quantitative SFA CFI}

Maximum physical workload achieved as a continuous parameter is not dependent on an educated guess, and as such, is likely more dependable than certain GPAQ items. This appears the most probable explanation for a mere trend towards a relation between (some) GPAQ items and SFA CFI versus an actual significant and direct correlation between maximum workload and SFA CFI. However, figure 5 illustrates that approximately $92 \%$ of the SFA CFI variability was explained by parameters other than varying maximum workload. The key factor contributing to the 'noise' in the relation between maximum workload and SFA CFI is the time lag between the exercise test and SFA collateral function measurement. On average, the exercise test had been performed 90 days before the invasive exam with SFA CFI, and the time interval between the two events ranged from 5 years before to 1.5 years after the invasive exam. As a theoretical alternative for the scatter between the two variables, SFA CFI did not accurately reflect quantitative SFA collateral function.

\section{Validation of quantitative SFA CFI}

This hypothesis was tested in $80 \%$ of the study patients by simultaneous comparison of SFA CFI with an independent, quantitative method, transcutaneous $\mathrm{PO}_{2}$ index. It was falsified by documenting a tight and direct association between the two independent methods. Bland-Altman bias between the two methods was +0.065 , and the limits of agreement -0.18 and +0.31 . The trend to underestimate CFI with higher collateral function values might serve as an explanation for the flat rather than more steep relation between maximum workload and SFA CFI. Conversely, the $\mathrm{PO}_{2}$ method, though employed and validated in the past, ${ }^{25-27}$ may have technical shortcomings challenging its reference status. Notwithstanding, there was also (at least trend-wise; $\mathrm{p}=0.12$ ) a direct association between maximum workload and transcutaneous $\mathrm{PO}_{2}$ index.

\section{Study limitations}

The study limitations alluded to and discussed above were the method of GPAQ data acquisition by unassisted written inquiry, and the time lag between obtaining physical exercise data (GPAQ or exercise test) and SFA CFI measurement. Both limiting factors may have influenced the results against the study hypothesis, thus conserving the null hypothesis of no relation between these potential predictors and SFA collateral function.

Acknowledgements The authors thank Hélène Steck, RN and Raphael Grossenbacher, RN for their valuable work in patient recruitment, and expert technical assistance in data acquisition.

Contributors MS and DS were responsible for data collection, data analysis, drafting the manuscript. CS was responsible for study conception, data collection, invasive procedures, data analysis and writing the manuscript.

Funding This study was supported by grants from the Swiss National Science Foundation for research (grant \#3200B_141030/1 to CS), and from the Novartis Foundation for Medical-Biological Research grant \#14B055 (to MS and CS).

Competing interests None declared.

Ethics approval Kantonale Ethikkommission Bern.

Provenance and peer review Not commissioned; externally peer reviewed.

Data sharing statement No additional data are available.

Open Access This is an Open Access article distributed in accordance with the Creative Commons Attribution Non Commercial (CC BY-NC 4.0) license, which permits others to distribute, remix, adapt, build upon this work noncommercially, and license their derivative works on different terms, provided the original work is properly cited and the use is non-commercial. See: http:// creativecommons.org/licenses/by-nc/4.0/

\section{REFERENCES}

1. WHO. Global status report on noncommunicable diseases 2010 . Geneva: World health organization, 2011.

2. Nehler MR, Duval S, Diao L, et al. Epidemiology of peripheral arterial disease and critical limb ischemia in an insured national population. J Vasc Surg 2014;60:686-95.e2. 
3. Ziegler MA, Distasi MR, Bills RG, et al. Marvels, mysteries, and misconceptions of vascular compensation to peripheral artery occlusion. Microcirculation 2010;17:3-20.

4. Traupe T, Ortmann J, Stoller M, et al. Direct quantitative assessment of the peripheral artery collateral circulation in patients undergoing angiography. Circulation 2013;128:737-44.

5. Schirmer SH, Millenaar DN, Werner $\mathrm{C}$, et al. Exercise promotes collateral artery growth mediated by monocytic nitric oxide. Arterioscler Thromb Vasc Biol 2015;35:1862-71.

6. Gardner AW, Poehlman ET. Exercise rehabilitation programs for the treatment of claudication pain. A meta-analysis. JAMA 1995;274:975-80.

7. McDermott MM, Ades P, Guralnik JM, et al. Treadmill exercise and resistance training in patients with peripheral arterial disease with and without intermittent claudication: a randomized controlled trial. JAMA 2009;301:165-74.

8. McDermott MM, Liu K, Guralnik JM, et al. Home-based walking exercise intervention in peripheral artery disease: a randomized clinical trial. JAMA 2013;310:57-65.

9. Lane $\mathrm{R}$, Ellis $\mathrm{B}$, Watson $\mathrm{L}$ and, et al Exercise for intermittent claudication. Cochrane Database Syst Rev 2014;(7): CD000990.

10. McDermott MM, Carroll TJ, Kibbe M, et al. Proximal superficial femoral artery occlusion, collateral vessels, and walking performance in peripheral artery disease. JACC Cardiovasc Imaging 2013;6:687-94.

11. Cleland CL, Hunter RF, Kee F, et al. Validity of the global physical activity questionnaire (GPAQ) in assessing levels and change in moderate-vigorous physical activity and sedentary behaviour. $B M C$ Public Health 2014;14:1255.

12. Seiler C, Fleisch M, Garachemani AR and, et al Coronary collateral quantitation in patients with coronary artery disease using intravascular flow velocity or pressure measurements. J Am Coll Cardiol 1998;32:1272-9.

13. Matsuo $\mathrm{H}$, Watanabe $\mathrm{S}$, Kadosaki $\mathrm{T}$, et al. Validation of collatera fractional flow reserve by myocardial perfusion imaging. Circulation 2002;105:1060-5.

14. Vogel R, Zbinden R, Indermühle $A$, et al. Collateral-flow measurements in humans by myocardial contrast echocardiography: validation of coronary pressure-derived collateral-flow assessment. Eur Heart J 2006;27:157-65.
15. WHO. WHO STEPS surveillance manual: the WHO STEPwise approach to chronic disease risk factor surveillance. World Health Organization, 2005.

16. Bull FC, Maslin TS, Armstrong T. Global physical activity questionnaire (GPAQ): nine country reliability and validity study. $J$ Phys Act Health 2009;6:790-804.

17. Wallmann-Sperlich B and Froboese I. Physical activity during work, transport and leisure in Germany-prevalence and socio-demographic correlates. PLOS ONE 2014;9:e112333.

18. Sobieszczyk PS, Beckman JA. Intervention or exercise? The answer is yes!. J Am Coll Cardiol 2015;65:1010-12.

19. Rooke TW, Hirsch AT, Misra S, et al. Management of patients with peripheral artery disease (compilation of 2005 and 2011 ACCF/AHA Guideline Recommendations): a report of the American College of Cardiology Foundation/American Heart Association Task Force on Practice Guidelines. J Am Coll Cardiol 2013;61:1555-70.

20. Murphy TP, Cutlip DE, Regensteiner JG, et al. Supervised exercise, stent revascularization, or medical therapy for claudication due to aortoiliac peripheral artery disease: the CLEVER study. J Am Coll Cardiol 2015;65:999-1009.

21. Fakhry F, Spronk S, van der Laan L, et al. Endovascular revascularization and supervised exercise for peripheral artery disease and intermittent claudication: a randomized clinical trial. JAMA 2015;314:1936-44.

22. Keeling AN, Carroll TJ, McDermott MM, et al. Clinical correlates of size and number of collateral vessels in peripheral artery disease. Vasc Med 2012;17:223-30.

23. Mays RJ, Casserly IP, Kohrt WM, et al. Assessment of functional status and quality of life in claudication. J Vasc Surg 2011;53:1410-21.

24. Senti S, Fleisch M, Billinger M, et al. Long-term physical exercise and quantitatively assessed human coronary collateral circulation. J Am Coll Cardiol 1998;32:49-56.

25. Shepherd JT. The effect of acute occlusion of the femoral artery on the blood supply to the calf of the leg before and after release of sympathetic vasomotor tone. Clin Sci 1950;9:355-65.

26. Frau G. Transcutaneous PO2 response to transient arterial occlusion in peripheral vascular disease detected by heating power oximeter. Angiology 2001;52:851-7.

27. Salman M, Glantzounis GK, Yang W, et al. Measurement of critical lower limb tissue hypoxia by coupling chemical and optical techniques. Clin Sci (Lond) 2005;108:159-65. 